University of Nebraska - Lincoln

DigitalCommons@University of Nebraska - Lincoln

Ralph Skomski Publications

Research Papers in Physics and Astronomy

September 1996

\title{
The Itinerant Limit of Metallic Anisotropy
}

Ralph Skomski

University of Nebraska-Lincoln, rskomski2@unl.edu

Follow this and additional works at: https://digitalcommons.unl.edu/physicsskomski

Part of the Physics Commons

Skomski, Ralph, "The Itinerant Limit of Metallic Anisotropy" (1996). Ralph Skomski Publications. 11. https://digitalcommons.unl.edu/physicsskomski/11

This Article is brought to you for free and open access by the Research Papers in Physics and Astronomy at DigitalCommons@University of Nebraska - Lincoln. It has been accepted for inclusion in Ralph Skomski Publications by an authorized administrator of DigitalCommons@University of Nebraska - Lincoln. 


\title{
The Itinerant Limit of Metallic Anisotropy
}

\author{
Ralph Skomski
}

\author{
Max-Planck-Institut für Mikrostrukturphysik, Weinberg 2, 06120 Halle, Germany
}

\begin{abstract}
The anisotropy contribution of itinerant electrons confined to a quantum-well potential is calculated and compared with the quasi-ionic contribution due to virtually bound electrons. The easy magnetization direction of the non-L-S quantum-well electrons lies in the plane, and the magnitude of the anisotropy, maximally of order $10 \mathrm{~J} / \mathrm{m}^{3}$, is too weak to explain anisotropies encountered in practice. This means that itinerant $3 d$ anisotropy in bulk materials and thin films is associated with Hund's-rules-type ionic contributions.
\end{abstract}

\section{INTRODUCTION}

Magnetic anisotropy caused by itinerant electrons in bulk materials and thin films has attracted considerable attention in recent years. Examples are the phenomenon of pcrpendicular thin-film anisotropy relevant to magnetooptical recording [1] and the $3 \mathrm{~d}$ contribution to the magnetocrystalline anisotropy of permanent magnets such as $\mathrm{SmCo}_{5}$ and $\mathrm{Nd}_{2} \mathrm{Fe}_{14} \mathrm{~B}[2,3]$. Apart from a magnetostatic contribution of order $\mu_{\mathrm{o}} \mathrm{M}_{\mathrm{S}}^{2}$, magnetic anisotropy is of magneto-crystalline origin and involves relativistic spin-orbit coupling and electrostatic crystal-field interaction. Essentially, the orbital motion of the electrons is influenced by the electrostatic potential of the crystal, and spin-orbit interaction couples the orbital motion of the electrons to spin and magnetization. However, the detailed anisotropy mechanism depends on the strengths of spin-orbit coupling and crystal-field interaction as well as on the degree of localization of the magnetic electrons.

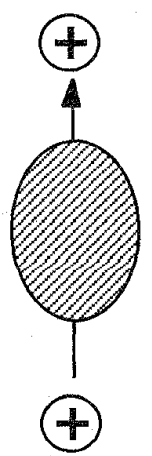

PROLATE

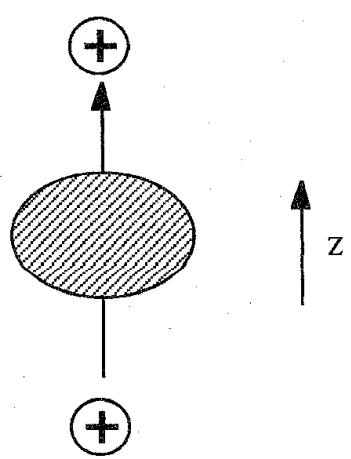

OBLATE
Fig. 1. Ionic prolaticity and anisotropy. In this example, prolate and oblate ions yield easy anisotropies parallel and perpendicular to the $z$ axis, respectively.
Magnetocrystalline anisotropy in modern rare-earth permanent magnets such as $\mathrm{SmCO}_{5}$ [4], $\mathrm{Nd}_{2} \mathrm{Fe}_{14} \mathrm{~B}$ [2], and $\mathrm{Sm}_{2} \mathrm{Fe}_{17} \mathrm{~N}_{3}$ [5] largely originates from the rare-earth sublattice. In spite of the comparatively low volume fraction of the rare earths, typical rare-earth anisotropy contributions are of order $10 \mathrm{MJ} / \mathrm{m}^{3}$ in these intermetallics $[4,6,7]$. Tripositive rare-earth ions are reasonably well described by Hund's rules, so that rare-earth anisotropy may be interpreted in terms of the electrostatic interaction of well-localized $4 f$ electron shells with the crystal environment [6-8]. Figure 1 shows prolate and oblate $4[$ charge distributions in a crystal environment symbolized by positive charges above and below the ion. Since there, is a firm coupling between the 41 charge cloud and the magnetic moment, the preferred magnetization direction is obtained by minimizing the electrostatic energy of the ion.

Compared to rare-earth anisotropy contributions, the anisotropy caused by itinerant d electrons tends to be rather low. However, anisotropies of order $1 \mathrm{MJ} / \mathrm{m}^{3}$ are observed in thin films [1] as well as in layered intermetallics such as Y $\mathrm{CO}_{5}$ and $\mathrm{PtCo}$ [9]. A good example is the L1, compound $\mathrm{PtCo}$, which can be regarded as a tetragonally distorted fec derivate consisting of alternating layers of magnetic (3d) and nonmagnetic elements. This makes it possible to treat itinerant interface, surface, and bulk anisotropies on a common basis.

$3 \mathrm{~d}$ anisotropy in metals is characterized by two basic features. First, as in nonmetallic magnets the orbital moment of the $3 \mathrm{~d}$ electrons is largely suppressed by the crystal field. This quenching does not only affect the spontaneous magnetization but also reduces the magnetocrcrystalline anisotropy. Secondly, metallic $3 \mathrm{~d}$ electrons are itinerant, and the question arises to what extent the ionic anisotropy mechanism survives the delocalization of the $3 \mathrm{~d}$ electrons. From the point of view of band-structure theory, anisotropy produced by itinerant $3 \mathrm{~d}$ electrons can be regarded as a higher-order perturbation, and reasonable anisotropy predictions have been made in a number of cases [10-13]. Essentially, one includes spin-orbit interaction in addition to the energy terms appearing in the Stoner theory or in spin-polarized band-structure calculations. Since the charge density of the metallic $3 \mathrm{~d}$ electrons remains, in some sense, reminiscent of that of free ions, band-structure calculations mix ionic and itinerant features. For instance, in the limit of weakly overlapping tight-binding orbitals the problem retains its ionic character, although the matrix elements between different orbitals are now wave-vector dependent [10]. Here we discuss the nature of itinerant anisotropy in terms of analytical approaches. 


\section{IONIC 3dA NISOTROPY}

Experimental evidence in favour of the ionic picture of itinerant $3 \mathrm{~d}$ anisotropy comes from the fact that iron and cobalt tend to exibit opposite anisotropy contributions in isostructural intermetallics $[6,14]$. For instance, roomtemperature bec iron has $\mathrm{K}_{1}=42 \mathrm{~kJ} / \mathrm{m}^{3}$, whereas iron-cobalt alloys with $30 \mathrm{wt} . \%$ and $70 \mathrm{wt} \%$ cobalt exhibit $\mathrm{K}_{1}=10$ $\mathrm{kJ} / \mathrm{m}^{3}$ and $\mathrm{K}_{1}=-43 \mathrm{~kJ} / \mathrm{m}^{3}$, respectively [15]. Another example are the room-temperature $\mathrm{Y}_{2} \mathrm{Fe}_{14} \mathrm{~B}$ and $\mathrm{Y}_{2} \mathrm{Co} 14 \mathrm{~B}$ anisotropies $\mathrm{K}_{1}=1.1 \mathrm{MJ} / \mathrm{m}^{3}$ and $\mathrm{K}_{1}=-1.2 \mathrm{MJ} / \mathrm{m}^{3}$, respectively. Both magnetization measurements and bandstructure calculations indicate that the quasi-ionic states of $3 \mathrm{~d}$ atoms in metals are close to $\mathrm{T}^{1+}$ configurations, i.e. one of the two $4 \mathrm{~s}$ electrons is accommodated in the $3 \mathrm{~d}$ band $[14$, 16]. Examining the $3 d$ Stevens coefficients [17], which determine the shape of the ionic charge distribution, one finds that both $\alpha=\theta_{2}$ and $\beta=\theta_{4}$ change sign between the $3 \mathrm{~d}^{7}\left(\mathrm{Fe}^{+}\right)$and $3 \mathrm{~d}^{8}\left(\mathrm{Co}^{+}\right)$configurations [14]. Thus, if there is a partial survival of the ionic multipole moments on band formation, then the shape difference between (oblate) iron and (prolate) cobalt ions gives rise to opposite anisotropy contributions in a given crystal field. A pictorial explanation is that the $3 \mathrm{~d}$ electrons travelling through the lattice are temporarily captured by the ionic cores of the $3 \mathrm{~d}$ atoms, where they benefit from the ionic spin orbit coupling.

To quantify this quasi-ionic behaviour we recall that the shape of Hund's-rule ground-state electron clouds is given by the electrostatic quadrupole moment [17]

$$
Q_{2}=\theta_{2}<\mathrm{r}^{2}>_{\mathrm{n} 1}\left(3 \mathrm{~J}^{2}-\mathrm{J}(\mathrm{J}+1)\right)
$$

In the case of $3 \mathrm{~d}$ electrons, spin and orbital moment are largely decoupled, so that $J$ has to be replaced by $L$. Introducing the number $n$ ' of electrons in the second half of the $3 \mathrm{~d}$ shell we then obtain, after short calculation,

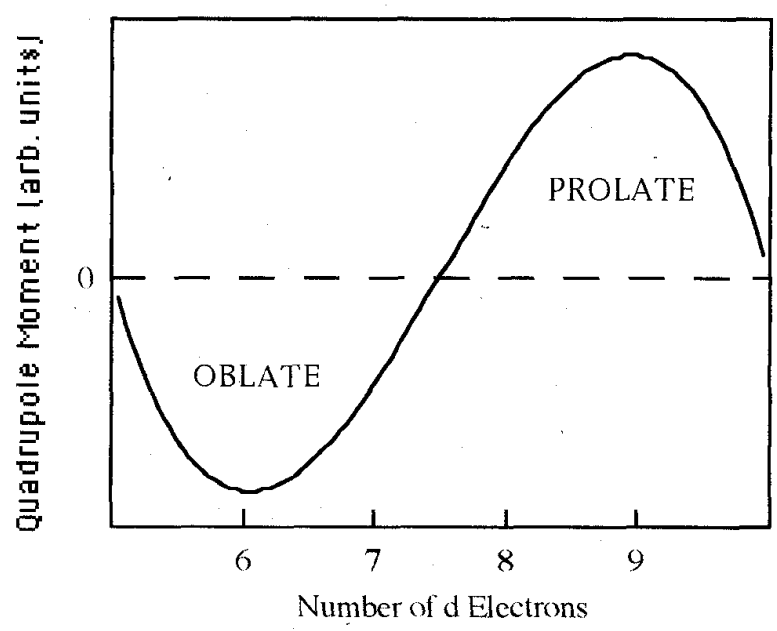

Fig. 2. Ionic 3d quadrupole moments $Q_{2}$. In a given lattice environment, the first anisotropy constant $K_{1}$ is proportional to $Q_{2}$.

$$
\mathrm{Q}_{2}=-\frac{\mathrm{n}^{\prime}}{21}\left(5-\mathrm{n}^{\prime}\right)\left(5-2 \mathrm{n}^{\prime}\right)<\mathrm{r}^{2}>_{\mathrm{n} 1}
$$

Figure 2 shows this equation as a function of the total number of d electrons, $n=5+n^{\prime}$. Note, however, that $E q$. (1) cannot be used to make quantitative predictions of $\mathrm{K}_{1}$, since only part of the free-ion multipole moment Q2 remains unquenched.

We see that the $3 \mathrm{~d}$ anisotropy changes sign at $n=7.5$ and reaches a maximum at $n=8.943$. Since the moment of strong ferromagnets is given by the d-band filling, equation (2) predicts the $3 \mathrm{~d}$ anisotropy to be most pronounced if there are 1.057 holes in the spin-down band. This value is indeed compatible with the behaviour of $\mathrm{Co} / \mathrm{Ni}$ multilayers, where experiment and detailed band-structure calculations indicate perpendicular anisotropy between about 9 and 10 valence electrons per atom [11].

\section{ITINERANT 3d A NISOTROPY}

Consider the anisotropy of an electron gas confined to a set of $x$-y planes (Fig. 3). The atomic potential in real solids is more or less spherical around the atomic nuclei (Fig. 3a), although the overlap of the atomic wave functions gives rise to band-structure corrections. In the opposite limit of free electrons it is suitable to start from a quantum-well potential where the ionic cores are neglected (Fig. 3b). Since the usual L-S-type spin-orbit interaction is based on spherical potentials, one has to start from the more general FoldyWouthuysen-transformed Dirac equation [18]

$$
H_{m n}=\frac{\hat{p}^{2}}{2 m} \delta_{m n}+V(\mathbf{r}) \delta_{m n}+\frac{\hbar}{4 m^{2} c^{2}} \boldsymbol{\sigma}_{m n} \cdot(\nabla V \times \boldsymbol{p})
$$

where the indices refer to the two-component spin wave functions $\Psi_{m}(\mathbf{r})=\left(\Psi_{1}, \Psi_{2}\right), \delta_{m n}$ is the unit matrix in spin space, and $\mathbf{o}_{\mathrm{mn}}$ denotes the Pauli spin matrices.

Exchange ficlds in $3 \mathrm{~d}$ ferromagnets are generally much larger than anisotropy fields, so that there is a well-defined quantization axis along the magnetization direction. The spin operator $\boldsymbol{\sigma}_{\mathrm{mn}}$ can then be replaced by its eigenvalue - $\mathbf{M} / \mathrm{M}_{\mathrm{O}} \delta_{\mathrm{mn}}$

$E \Psi=\frac{\hat{p}^{2}}{2} \Psi+V(\mathbf{r}) \Psi-\frac{h}{4 m^{2} c^{2} M_{o}}(\mathbf{M} \times \nabla V) \cdot \hat{\mathbf{p}} \Psi$

Note that paramagnetic metals, which do not exhibit magnetocrystalline anisotropy, and actinide or $5 \mathrm{f}$ metals, where the spin-orbit coupling is extremely strong, are not considered here. Without loss of generality we can put $\mathbf{M}=$ $M_{\mathrm{o}}\left(\sin \theta \mathbf{e}_{\mathrm{x}}+\cos \theta \mathbf{e}_{\mathrm{z}}\right)$ in Eq. (4), so that

$$
E \Psi=-\frac{h^{2}}{2 m} \nabla^{2} \Psi+V \Psi-i b \frac{\partial V}{\partial z} \sin \theta \frac{\partial \Psi}{\partial y}
$$

where $b=h^{2} / 4 m^{2} c^{2}$. A further simplification is achieved by the ansatz $\Psi=\exp \left(\mathrm{ikx}_{\mathrm{x}} \mathrm{x}+\mathrm{iky} \mathrm{y}\right) \Phi(\mathrm{z})$. Putting

$$
E=\frac{h^{2}}{2 m}\left(k_{x}^{2}+k_{y}^{2}\right)+E_{z}
$$

then yields

$$
\mathrm{E}_{z} \Phi=-\frac{h^{2}}{2 \mathrm{~m}} \frac{\partial^{2} \Phi}{\partial z^{2}}+\left(\mathrm{V}-\mathrm{k}_{\mathrm{y}} \mathrm{b} \frac{\partial \mathrm{V}}{\partial z} \sin \theta\right) \Phi
$$




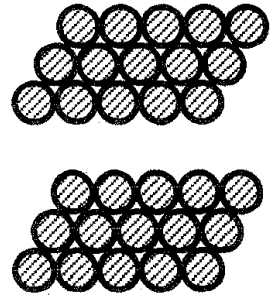

$\mathbf{a}$
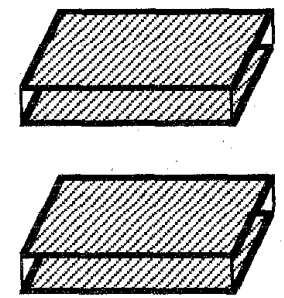

$\mathbf{b}$
Fig. 3. Layered array of magnetic atoms (schematic): a true potential and $\mathbf{b}$ quantum-well potential.

In the case of a periodic potential $V(z)$ this equation leads to a one-dimensional tight-binding band-structure problem in $\mathrm{z}$ direction and a two-dimensional free-electron-type bandstructure problem in the $\mathrm{x}$ and $\mathrm{y}$ directions. If electron hopping in $\mathrm{z}$ direction is negligible we can restrict ourselves to a single plane centered at $z=0$, so that series expansion yields the approximate potential $\mathrm{V}(\mathrm{z})=\mathrm{V}_{\mathrm{O}} \mathrm{Z}^{2 / 2}$. Thus,

$$
E_{z} \Phi=-\frac{h^{2}}{2 m} \frac{\partial^{2} \Phi}{\partial z^{2}}+v_{o}\left(\frac{z^{2}}{2}-k_{y} b z \sin \theta\right) \Phi
$$

which can be rewritten as

$$
\begin{aligned}
E_{Z} \Phi=-\frac{h^{2}}{2 m} \frac{\partial^{2} \Phi}{\partial z^{2}}+ & \frac{V_{o}}{2}\left(z-k_{y} b \sin \theta\right)^{2} \Phi \\
& -\frac{V_{o}}{2} k_{y}{ }^{2} b^{2} \sin ^{2} \theta \Phi
\end{aligned}
$$

This is the wave equation for a one-dimensional harmonic oscillator whose center of gravity is given by $z_{0}=k_{y} b \sin$ $\theta$. Since the energy of a harmonic oscillator does not depend on the center of gravity, the anisotropy energy equals the last term in Eq. (9). Introducing the average squared oscillation amplitude $\mathrm{a}^{2}=\left\langle\left(\mathrm{z}-\mathrm{z}_{\mathrm{O}}\right)^{2}\right\rangle$ and the ground-state energy $\mathrm{E}_{\mathrm{O}}=$ $\mathrm{h}^{2} /\left(4 \mathrm{ma}^{2}\right)$ we obtain the anisotropy energy

$$
\Delta E(\mathbf{k})=-\frac{E_{0}}{2} k_{p}^{2} a^{2} \frac{E_{0}^{2}}{m^{2} c^{4}} \sin ^{2} \theta
$$

Here the in-plane wave-vector component $\mathrm{k}_{\mathrm{p}}$ equals $\mathrm{k}=|\mathbf{k}|$, since we have neglected interplane hopping.

Examination of (10) shows that the preferred magnetization direction lies in the $x-y$ plane, so that the electron confinement Fig. 3b leads to easy-plane anisotropy. Note that this easy-plane anisotropy is not restricted to quadratic potentials but also occurs for other in-plane energy minima.

The total anisotropy energy is obtained by averaging over all electronic $\mathrm{k}$-space vectors. Up to a factor of order one, the averaging amounts to the replacement of $\mathrm{kp}^{2}$ in (10) by $\mathrm{kF}^{2}$. Estimating the anisotropy by taking $\mathrm{kF}=1 / \mathrm{a}$ and $\mathrm{E}_{\mathrm{O}}=10$ $\mathrm{eV}$ yields the quite small anisotropy energy $\Delta E=-0.022$ $\mathrm{mK}$, which has to be compared to experimental anisotropy energies of order $1 \mathrm{~K}$.

\section{DISCUSSION AND CONCLUSIONS}

The predicted quantum-well anisotropies are non-zero but very small, so it is difficult to measure or simulate these contributions in real materials. This is in agreement with the experimental and theoretical arguments presented in Sections I and II, which indicate that quasi-ionic contributions are sufficient to explain observed anisotropy trends. On the other hand, the existence of quantum-well anisotropy shows that L-S coupling and Coulomb attraction associated with atomic nuclei of charge $Z \mathrm{e}$ are not necessary to produce magnetocrystalline anisotropy.

In conclusion, the non-L-S anisotropy associated with the free motion of itinerant electrons is non-zero but too weak to explain experimental anisotropy constants. Itinerant anisotropy in $3 \mathrm{~d}$ metals is largely due to temporarily localized (virtually bound) states having much in common with truely localized orbitals.

\section{A CKNOWLEGDEMENTS}

Many thanks to J. M. D. Coey, J. Kirschner and Y. Millev for stimulating discussions. Part of this work has been performed at Physies Department, Trinity College, Dublin.

\section{REFERENCES}

[1] U. Gradmann, in: Landolt-Börnstein, vol. $111-19 \mathrm{~g}$, Ed.: H. P. J. Wijn, Springer, Berlin 1988.

[2] M. Sagawa, S. Fujimura, N. Togawa, H. Yamamoto, and Y. Matsuura, J. Appl. Phys., vol. 55, p. 2083, 1984.

[3] J. E. Evetts, ed., Concise Encyclopedia of Magnetic and Superconducting Materials, Pergamon, Oxford, 1992.

[4] K. H. J. Buschow, Rep. Prog. Phys, vol. 40, p. 1179 , 1977.

[5] J. M. D. Coey and H. Sun, J. Magn. Magn. Mater., vol. 87, p. L251, 1990.

[6] J. F. Herbst, Rev. Mod. Phys., vol. 63, p. 819, 1991.

[7] J. M. D. Coey, in: Science and Technology of Nanostructured Magnetic Materials, Eds. G. C. Hadjipanayis and G. A. Prinz, Plenum, New York 1991, p. 439.

[8] R. Skomski, in: Interstitial Intermetallic Alloys, Eds.: F. Grandjean, G. J. Long, and K. H. J. Buschow, Kluwer, Dordrecht, 1995, p. 561.

[9] N. N. Shegoleva, L. M. Nagat, and Ya. T. Zhur, Fiz. Met. Metalloved., vol. 34, p. 663, 1972.

[10] H. Brooks, Phys. Rev., vol. 58, p. 909, 1940.

[11] G. H. O. Daalderop, P. J. Kelly, and F. J. A. den Boeder, Phys. Rev. Lett., vol. 68, p. 682, 1992.

[12] R. H. Victora and J. M. McLaren, Phys. Rev. B, vol. 47, p. $11583,1993$.

[13] A. J. Freeman, R. Wu, and D. S. Wang, in: Proc. ISPMM, Seoul 1995, p. 845.

[14] J. J. M. Franse, F. E. Kayzel, N. P. Thuy, J. Magn. Magn. Mater., vol. 129, p. 26, 1994.

[15] R. M. Bozorth, Ferromagnetism, van Nostrand, Princeton, New Jersey, 1951.

[16] J. Crangle, Solid-State Magnetism, Arnold, London 1991.

[17] M. T. Hutchings, Solid State Phys., vol. 16, p. 227 , 1964.

[18] W. Jones and N. H. March, Theoretical Solid State Physics I, Wiley \& Sons, London 1973. 